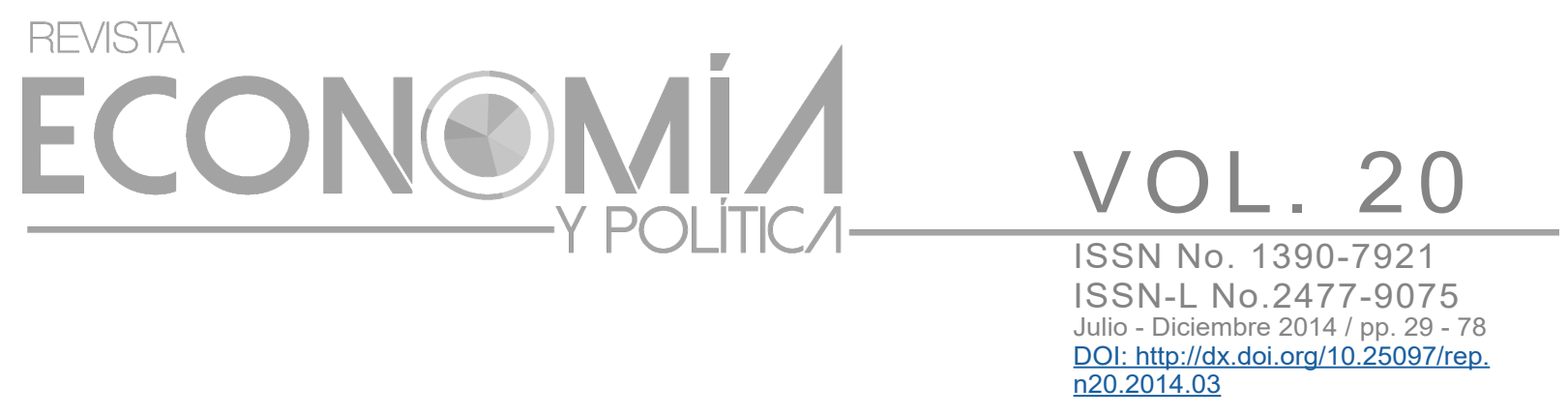

\title{
METODOLOGÍAS PARTICIPATIVAS CON ENFOQUE INTEGRADOR DESDE LA COMPLEJIDAD
}

\section{PARTICIPATORY METHODOLOGIES WITH INTEGRATIVE APPROACH FROM THE COMPLEXITY}

Gabriela Álava Atiencie

Universidad de Cuenca

gabriela.alava@ucuenca.edu.ec
Elvis Orellana Espinoza

Universidad de Cuenca

elvis.orellana@ucuenca.edu.ec

\begin{abstract}
The article presents a scientific literature review of proposals that have emerged with the epistemological dominance of positivism, reductionist and mechanistic worldview. These alternatives are:

The complexity theory that exceeds the reductionist, mechanistic and dichotomous thinking, and allows to analyze the reality from different perspectives, approaches and knowledge, and catalyzes the transformation and changing situations.

The systems theory, which proposed that the change actually comes from the selforganizing and emergent systems.

The Participatory Methodologies raising various techniques that promote dialogue between academic knowledge and social knowledge (popular).

Integrative approaches and decolonization thoughts that transcend and go beyond positivism as modes of knowledge production based on subject-subject relations, rather than subject-object relations.
\end{abstract}

Key words: Complexity, decolonization, emergent systems, methodology, participatory methodologies, socio- praxis.

\section{RESUMEN}

El artículo presenta una revisión literaria científica de propuestas que han surgido ante el predominio epistemológico del positivismo y de una visión reduccionista y mecanicista del mundo. Estas alternativas son:

- La teoría de la complejidad que supera el pensamiento reduccionista, mecanicista y dicotómico; ya que permite analizar la realidad desde diversas perspectivas, enfoques y saberes, y al mismo tiempo cataliza la transformación y el cambio de situaciones.

- La teoría de sistemas en la que se propone que el cambio de la realidad viene desde los sistemas emergentes o auto organizados. 
- Las Metodologías participativas que plantean diversas técnicas que promueven el diálogo entre el saber académico y el saber social (popular).

Enfoques integradores y descolonizadores del pensamiento que trascienden y desbordan el positivismo como modos de producción de conocimiento basados en relaciones sujeto-sujeto, más que en sujeto-objeto.

\section{Palabras clave: Complejidad, descolonización del pensamiento, sistemas} emergentes, metodología, metodologías participativas, socio-praxis.

\section{INTRODUCCIÓN}

El modo dominante de producción de conocimiento de las ciencias sociales ha sido el reduccionismo positivista, en donde se concibe a los seres humanos y a sus relaciones como objetos o como cosas; el positivismo tiene como meta la búsqueda de leyes universales que explican la naturaleza y el mundo. Por ejemplo, el enfoque positivista en las ciencias sociales busca las leyes universales que expliquen el comportamiento social.

En el campo de la investigación, el positivismo es aventajado, ya que se busca permanentemente la objetividad y la búsqueda de leyes científicas universales como si en las mismas no estuvieran presentes aspectos de poder como lo indica Tomás R. Villasante:

"En las universidades muchas veces se plantean las investigaciones como si no influyese el promotor (económico, administrativo, etc.) de la misma, o como si no influyesen los prejuicios de los propios técnicos en los diseños y en las interpretaciones, y como si con cualquier población diese lo mismo aplicar las metodologías, pues se da por supuesto (en la mayoría de los casos) que las poblaciones no tienen capacidad o estrategias propias para dar o negar información básicas. Pero para un análisis más fino de nuestras propias investigaciones conviene hacerse preguntas" (2009, p.63).

Los modos epistemológicos dominantes en ciencias sociales, como el positivismo, son parte de una colonialidad del saber, entendida como la pretensión de universalización de un conocimiento geográfica y culturalmente construido: 
"El conocimiento tiene una relación y forma parte integral de la construcción y organización del sistema-mundo moderno capitalista que, a la vez y todavía, es colonial. Es decir, la "historia" del conocimiento está marcada geo histórica, geo política y geo cultural; tiene valor, color y lugar "de origen". En América Latina esta geopolítica se evidencia sobre todo en el mantenimiento del eurocentrismo como perspectiva única, o al menos dominante, del conocimiento. Es una perspectiva presente en universidades, colegios, escuelas, que exalta la producción intelectual euroamericana como ciencia y conocimiento universales, relegando en forma oposicional el pensamiento del Sur -sea latinoamericano, africano o de los otros "sures" del planeta- al estatus de "saber localizado", negando el hecho de que el conocimiento producido en Europa y Estados Unidos también es local; su universalización al resto del mundo como algo obligatorio para todos es, en efecto, el problema central de la geo política del conocimiento y su legado colonial e imperial" (Walsh, 2009, p. 187).

Pero además la colonialidad del saber se la puede relacionar con lo planteado por Boaventura de Sousa Santos (2010: pp. 47 - 51) desde la Sociología de las Ausencias, es decir, con los cinco modos de producción de ausencia o no existencia: el ignorante, el retrasado, el inferior, el local o particular y el improductivo o estéril; frente a las realidades que cuentan como importantes: las científicas, avanzadas, superiores, globales o productivas.

- El ignorante: consiste en la transformación de la ciencia moderna y de la alta cultura en criterios únicos de verdad y de calidad estética, respectivamente.

- El retrasado: la idea según la cual la historia tiene sentido y dirección únicos y conocidos. La idea de que el tiempo es lineal y al frente del tiempo están los países centrales del sistema mundial y, junto a ellos, los conocimientos, las instituciones y las formas de sociabilidad que en ellos dominan.

- El inferior: consiste en la naturalización de las diferencias y la distribución de las poblaciones por categorías que naturalizan jerarquías. La clasificación racial y la clasificación sexual son las manifestaciones más señaladas de esta lógica.

- El local o particular: se trata de la escala que privilegia las entidades o realidades que se extienden su ámbito de acción por todo el globo y que, al 
hacerlo, adquieren la prerrogativa de designar entidades o realidades rivales como locales.

- El improductivo o estéril: consiste en que el crecimiento económico es un objeto racional incuestionable y, como tal, es incuestionable el criterio de productividad que mejor sirve a este objetivo. Ese criterio se aplica tanto a la naturaleza como al trabajo humano (Santos, 2010).

Por ejemplo, en la actualidad una forma de producción de ausencia a través de la ciencia moderna podrían ser los indicadores o criterios para crear los rankings universitarios internacionales; dichos indicadores son universales y no miran los contextos y especificidades de las diversas universidades del mundo. Los rankings son realizados principalmente por corporaciones transnacionales 0 universidades asentadas en Londres, Shangai, Seúl o California y que por casualidad no sean elaborados en Bolivia, Nigeria, Bangladesh u algún país o institución periférica del sistema mundial (Villavicencio, 2013).

Aquí valdría cuestionarse sobre: ¿cuáles son las corporaciones que generan dichos rankings mundiales?, ¿Con qué criterios?, ¿Con quiénes formula dichos criterios?, ¿Para qué modelo de sociedad están pensados esos criterios de evaluación? ¿Quiénes les financian?, ¿Cómo son los procesos evaluación?, ¿Tienen en cuenta los contextos sociales, históricos, culturales, económicos y políticos de las universidades?, etc.

Por otro lado, Josef Estermann realiza un análisis crítico de la universidad latinoamericana manifestando lo siguiente:

"Hasta mediados del siglo XVIII, las universidades latinoamericanas eran bastiones de la legitimación intelectual y "científica" del poder colonial, de la supuesta "superioridad" del espíritu europeo y de la fundamentación de la "inferioridad" de la raza "india" y "mestiza". Estaban en función casi exclusivamente de la elite criolla; es decir: eran centros de estudios de los españoles para los españoles, islas intelectuales en un mar agitado por olas de rebeldía y resistencia. Recién en la segunda mitad del siglo XVIII, ciertas universidades introducían poco a poco las ideas "seculares" y "modernos" de los racionalistas europeos ( $y$ en menor medida las de los empiristas británicos) y de 
los philosophes franceses de la llustración, con sus ideas revolucionarias de la igualdad, libertad y fraternidad de los seres humanos. Pero se trataba de un cambio meramente ideológico y formal, del mismo sujeto criollo, para luchar por la independencia política de la nueva oligarquía del yugo colonial, sin cambiar absolutamente nada referente a la inclusión de los/as mestizos/as y mucho menos de las y los indígenas en la producción del saber y el ejercicio del poder. La elite española era reemplazada por una elite criolla, con los mismos apellidos y los mismos títulos de propiedad. Durante la vida republicana, las universidades seguían fungiendo como instrumento de poder de la clase alta y blanca, incluyendo poco a poco a los mestizos (mistis) como "carne de cañón" intelectual para la defensa de sus intereses. Había sólo un relevo de intelectuales realistas por republicanos, a veces dentro de las mismas personas, pero sin alterar nada en la estructura colonial de las universidades y su mentalidad eurocéntrica y racista" (2009, p. 5).

Así, en la mayor parte de universidades latinoamericanas se ha hecho evidencia los modos de producción de ausencia o no existencia ya que, por ejemplo, en el caso de las ciencias sociales, humanidades y filosofía, en los cursos o asignaturas de los pensum de estudios se refleja la revisión de autores, textos, marco teóricos e investigaciones generadas en Europa o Estados Unidos. En psicología se revisa prioritariamente a Freud, Jung, Kurt Lewin; en sociología a Comte, Weber, Marx, etc.; en economía a David Ricardo, Adam Smith, entre otros. Sin embargo, se da poco espacio para el análisis y el estudio de Martín Baró, Maritza Montero, Pichon-Rivière, en psicología; a Fals Borda y González Casanova, en sociología; a Paulo Freire y Carlos Núñez, en educación; a Carlos Matus, Antonio Elizalde, Manfreed Max Neef, José Luis Coraggio o Luis Razeto en economía; a Humberto Maturana y Francisco Varela en sistemas complejos y autopoiesis; a Enrique Dussel en Filosofía; a Leonardo Boff en Teología y Ecología; a Arturo Escobar en Antropología, etc.

Otra forma de producir ausencia es la percepción como única cultura a las formas de vida de las clases altas occidentales y que desconocen la existencia de otras formas de vida como las de la cultura hindú, la cultura andina, la cultura maya, etc.; ya que 
cualquier saber que se recupere de ellas será catalogado por la ciencia moderna de "esotérico", "mágico" o "pensamiento mítico", etc.

El positivismo ha estado impregnado en la ciencia moderna como única forma de producción de conocimiento hasta mediados del siglo $\mathrm{XX}$, sin embargo, siempre han existido otras formas de generación del saber cómo la perspectiva historicista, la fenomenología, el existencialismo y posteriormente el constructivismo, por mencionar unas pocas.

Teniendo en cuenta el predominio epistemológico del positivismo y de una visión reduccionista y mecanicista del mundo también han surgido propuestas alternativas como las teorías de la complejidad, las teorías de sistemas, los enfoques ecológicos y los planteamientos desde las metodologías participativas a través de la socio-praxis.

Propuestas que trascienden y desbordan el positivismo. En la transducción:

"no sólo se trata de aprender de algunos aspectos superficiales de tales o cuales experiencias o planteamientos, sino de cambiar de plano, enfocar desde otras ópticas el conjunto de procesos... Transductores sociales serían redes que llevan, actuando como "dispositivos" (espejos y espirales), a unas transformaciones/saltos energéticos y de información y acción para vivificar los procesos en los que se implican. Estos es, por tanto, actuar con unos estilos en las redes y procesos que nos encontramos para hacerles más creativas" (Villasante, 2006, p. 36).

Hecho que analizaremos a continuación:

\section{COMPLEJIDAD Y SISTEMAS EMERGENTES}

Desde una visión ecológica Frijtof Capra (2006, pp. 25 - 27) afirma que:

"cuanto más estudiamos los principales problemas de nuestro tiempo, más nos percatamos de que no pueden ser entendidos aisladamente. Se trata de problemas sistémicos, lo que significa que están interconectados y son interdependientes... Asistimos a un cambio de paradigmas, no sólo de la ciencia, sino también en el más amplio contexto social...". 
Las perspectivas ecologistas y sistémicas de la realidad han trascendido los enfoques reduccionistas y las formas mecanicistas de ver el mundo. La perspectiva sistémica no separa el entorno para estudiarlo por disciplinas, sino que ven el todo sin reducirlo a sus partes, es decir, la relacionalidad y la interdependencia entre sus diversos componentes.

Los enfoques ecológicos y sistémicos de ver, estar y vivir el mundo no solo se los puede encontrar en la ciencia y en las instituciones académicas, porque también han estado presentes en la mayoría de sistemas culturales ancestrales; por ejemplo, en América Latina queda mucho por investigar sobre las cosmovisiones de los pueblos y nacionalidades indígenas.

En el caso ecuatoriano, el sumak kawsay ha sido un paradigma cultural y una cosmovisión integral que se ha puesto en debate en los últimos años pero que aún queda por ser indagado, explorado, investigado y recreado. Se lo presenta como una de los aportes más importantes de América Latina al igual que el Sumaq Qmaña de la cultura ancestral boliviana.

Estas nuevas formas de ver el mundo son importantes porque dan muestra de la evolución de la conciencia humana (Wilber, 2008), es decir, están caminando hacia una conciencia relacional, interdependiente y conectada con el todo.

Los paradigmas ecológicos y sistémicos permiten la recreación permanente de los saberes y el conocimiento desde las propias realidades y relaciones de los sujetos pero que al mismo tiempo pueden resultar prácticos y útiles a la gente. Gladys Adamson (2005, p. 144) afirma que "desde la práctica cotidiana se produce un saber que no se sabe que se sabe pero que emerge cuando se crean las condiciones y el dispositivo para ello".

Los planteamientos de Tomás R. Villasante $(2006,2009)$ desde las metodologías participativas y concretamente desde la socio-praxis apuntan a la puesta en práctica del saber y al cambio social desde las realidades de los sujetos. Las metodologías participativas apuntan a generar las condiciones para que surjan los saberes desde la vida cotidiana de los sujetos relacionales e interconectados.

Estos saberes cotidianos de los actores sociales de un contexto específico deben contar con las herramientas necesarias para que emerjan, por esto, las metodologías 
participativas apuntan a ser las catalizadoras de las complejidades, de los sistemas emergentes y de la transformación social.

Por otro lado, es importante no solamente quedarse en el aspecto racional de las metodologías participativas sino recuperar la integralidad de las mismas del ser humano teniendo en cuenta su corporalidad, sus emociones, sus sentires, sus vivencias, sus historias, sus territorios, sus culturas.

Además, es necesario evidenciar los sistemas emergentes o de los catalogados como "ignorantes", "retrasados", "inferiores", "locales" e "improductivos", como por ejemplo de los movimientos y colectivos indígenas latinoamericanos y sus aportes sobre la interculturalidad, de los movimientos vinculados al Foro Social Mundial y sus aportes sobre la democracia participativa, de los movimientos zapatistas y sus aportes sobre el buen gobierno, etc. Pero también las prácticas de Kerala sobre Planificación Participativa, de Porto Alegre sobre Presupuestos Participativos, por poner ejemplos internacionales.

En Ecuador están los casos de Salinas de Guaranda sobre Economía Social y Solidaria, de los cantones de Nabón y Cotacahi sobre Planificación Participativa, de la Comuna Agua Blanca en el Parque Nacional Machalilla en Manabí sobre Turismo Comunitario, por citar unos pocos.

\section{Replanteamiento cuanti-cuali desde lo participativo}

Diversos autores identifican en la construcción del conocimiento científico social, un proceso de al menos dos momentos metodológicos: estadístico y lingüístico. Ambos corresponden a las perspectivas metodológicas de investigación cuantitativa e investigación cualitativa, respectivamente. La perspectiva cualitativa de la investigación intenta acercarse a la realidad social a partir de motivaciones o posiciones no cuantitativas.

De este modo para profundizar en el sentido y alcance de estas dos metodologías es importante destacar algunos elementos históricos del desarrollo de la metodología 
Cuantitativa que surge a partir del siglo XIX y de la metodología Cualitativa que surge de manera posterior en el siglo $\mathrm{XX}$

Elementos históricos, metodológicos de la investigación Cuantitativa:

"Los fundamentos de la metodología cuantitativa podemos encontrarlos en el positivismo que surge en el primer tercio del siglo XIX como una reacción ante el empirismo que se dedicaba a recoger datos sin introducir los conocimientos más allá del campo de la observación. Alguno de los científicos de esta época dedicados a temas relacionados con las ciencias de la salud son Pasteur y Claude Bernard, siendo este último el que propuso la experimentación en medicina". (Álvarez R. 1996 citado por Pita Fernández, S., \& Pértegas Díaz, S., 2002, p.1).

"A principios del siglo XX, surge el neopositivismo o positivismo lógico siendo una de las aportaciones más importantes la inducción probabilística. La clave del positivismo lógico consiste en contrastar hipótesis probabilísticamente y en caso de ser aceptadas y demostradas en circunstancias distintas, a partir de ellas elaborar teorías generales. La estadística dispone de instrumentos cuantitativos para contrastar estas hipótesis y poder aceptarlas o rechazarlas con una seguridad determinada". (Pita Fernández, S., \& Pértegas Díaz, S. 2002, p.1).

Elementos históricos, metodológicos de la investigación Cualitativa:

Los métodos cualitativos tienen una historia en la investigación científica que se ha desarrollado más bien desde las ciencias sociales (Vera y Villalón) a partir del siglo XX. Miguel Valles (2000, pp. 14-16) en su texto "Técnicas Cualitativas de Investigación Social" cita los siguientes períodos históricos de su evolución de acuerdo a las teorías de Denzin y Lincoln:

1) El período tradicional es el momento de la etnografía clásica. "En este período, los investigadores cualitativitas escribían relatos "objetivos", colonizantes de las experiencias de campo, 
2) El período modernista se distingue por los numerosos esfuerzos de formalización de los métodos cualitativos esta etapa, conocida también como "la edad dorada del análisis cualitativo riguroso",

3) La fase de los "géneros desdibujados" o "borrosos" (blurred genres), en que "los límites entre las ciencias sociales y las humanidades se habían desdibujado". Los investigadores sociales buscan en la semiótica o en la hermenéutica nuevas teorías y métodos de análisis; y ,

4) Explica la crisis que enfrentan los investigadores sociales en este momento desdoblándola en una crisis de representación, por un lado, y de legitimación, por otro. La crisis de representación significa que se pone en cuestión una presunción que era básica en la investigación cualitativa anterior: la posibilidad misma de que el investigador (a) haga acopio directo de la experiencia vivida.

Así, uno de los debates más intensos en la investigación, se centra en las diferencias y bondades de los métodos de investigación cuantitativos y cualitativos (García, J. C., \& Martínez, M. R., 1996). En esta propuesta veremos cómo integrarlos y darles además un nuevo sentido participativo.

La estrategia metodológica cuantitativa está vinculada al enfoque positivista/colonialidad del saber y empirista de la ciencia que tiene una estrategia de números y métodos estadísticos. Se basan en medidas numéricas ciertos aspectos de los fenómenos parte de los casos concretos para llegar a una descripción general, pero en muchos de los casos para comprobar las hipótesis de las investigaciones. Entendiéndose por métodos de investigación cuantitativos a los diseños experimentales, cuasi-experimentales, la investigación por encuesta, los cuestionarios estandarizados, los registros estructurados de observación, las técnicas estadísticas de análisis de datos, etc. (García, J. C., \& Martínez, M. R. 1996)

La estrategia metodológica cualitativa, está vinculada al enfoque fenomenológico de la ciencia que tiene la estrategia de analizar significados, conceptos, metáforas, estudiar la relación que hay entre los hechos y el ámbito en que se hace presente esta realidad. Que conllevan a que el investigador utilice técnicas cualitativas como la 
entrevista, observación, observación participante, historias de vida, grupos de discusión o grupos focales que le permitan describir y sistematizar las experiencias en los casos objeto de análisis a partir de las narraciones; notas de campo; grabaciones; transcripciones de audio, vídeo y cassettes; registros escritos de todo tipo; fotografías, películas, entre otros utilizados para comprender la vida social desde una perspectiva holística que busca el análisis de un conjunto de eventos desde múltiples interacciones (Navarrete, 2004). Los roles que va desempeñando el investigador y los sujetos/elementos unidad de análisis del objeto de estudio son fruto de una definición y negociación progresiva. De esta forma, el investigador va asumiendo diferentes roles (investigador, participante) según su grado de involucramiento y participación en el estudio. Por su parte, los sujetos que forman parte de la investigación también van definiendo su papel según el grado en que proporcionan información (portero, informante clave, informante, ayudante o confidente). (Gómez, G. R., Flores, J. G., \& Jiménez, E. G., 1996).

Strauss indica que la diferencia fundamental entre ambos tipos de metodología estriba en que la investigación cuantitativa se centra en el estudio de las relaciones entre variables cuantificadas, mientras que la investigación cualitativa lo hace en contextos estructurales y situacionales (citado por Pita Fernández, S., \& Pértegas Díaz, S., 2002). Todo ello nos conlleva a visualizarlas como metodologías dominantes/predominantes que se han venido utilizando. La primera desde el positivismo lógico del estado a partir del siglo XIX y la segunda desde la fenomenología de la ciencia en la investigación social desde el siglo XX.

En el siguiente cuadro podemos observar de manera sintetizada cada una de las metodologías planteadas con sus especificidades, alcances y utilidades para la obtención de los resultados esperados en el estudio.

Cuadro Nro. 1

DIFERENCIAS ENTRE INVESTIGACIÓN CUANTITATIVA Y CUALITATIVA

INVESTIGACIÓN CUANTITATIVA

Basada en la inducción

probabilística del positivismo lógico Centrada en la fenomenología y comprensión 


\begin{tabular}{|l|l|}
\hline Medición penetrante y controlada & Observación naturista sin control \\
\hline Objetiva & Subjetiva \\
\hline Inferencias más allá de los datos & Inferencias de sus datos \\
\hline $\begin{array}{l}\text { Confirmatoria, inferencial, } \\
\text { deductiva }\end{array}$ & Exploratoria, inductiva, descriptiva \\
\hline Orientada al resultado & Orientada al proceso \\
\hline Datos "sólidos y repetibles" & Datos "ricos y profundos" \\
\hline Generalizable & No Generalizable \\
\hline Particularista & Holista \\
\hline Realidad estática & Realidad dinámica \\
\hline
\end{tabular}

Fuente: Artículo sobre "Investigación Cuantitativa y Cualitativa" pág. 2

Autores: Pita Fernández, S., \& Pértegas Díaz, S. (2002)

Las calificaciones de "objetiva" o "subjetiva" de este cuadro son tal como se consideran a sí mismas estas metodologías. Al igual que considerarse "medición penetrante y controlada", o bien "observación naturista sin control", que muchos otros científicos sociales pondrían en duda, por la simple constatación de como suelen operar muchas de estas metodologías en las prácticas reales. Pero valga el cuadro para poner un ejemplo del tipo de discusiones que existen entre cuantitativos y cualitativos.

Los estándares de calidad para los estudios cuantitativos están muy definidos y son conocidos universalmente pero este no es el caso para los estudios cualitativos. Sin embargo, existen algunos criterios que permiten evaluar el rigor y la calidad científica de los estudios cualitativos y sobre los cuales hay acuerdo parcial. Estos criterios son:

- La credibilidad,

- La auditabilidad o confiabilidad; y,

- La transferibilidad o aplicabilidad.

La credibilidad se logra cuando los hallazgos del estudio son reconocidos por las personas que participaron y por aquellas que han experimentado o estado en contacto con el fenómeno investigado. La confiabilidad se refiere a la neutralidad/imparcialidad que se logra cuando otros investigadores pueden seguir las interpretaciones y análisis 
de la información obtenida por el investigador original y llegar a hallazgos similares. La transferibilidad consiste en la posibilidad de trasladar y utilizar los resultados en otros contextos o grupos. (Castillo E., Vázquez M. 2003).

Desde este punto de vista resulta interesante analizar las ventajas e inconvenientes que presentan las dos metodologías y las preguntas cruzadas que se lanzan unos a otros:

Cuadro Nro. 2

VENTAJAS E INCONVENIENTES

DE LOS MÉTODOS CUANTITATIVOS VS. LOS MÉTODOS CUALITATIVOS

\begin{tabular}{|l|l|}
\hline \multicolumn{1}{|c|}{ MÉTODOS CUANTITATIVOS } & \multicolumn{1}{c|}{ MÉTODOS CUALITATIVOS } \\
\hline $\begin{array}{l}\text { Propensión a "servirse de" los } \\
\text { sujetos del estudio. }\end{array}$ & $\begin{array}{l}\text { Propensión a "comunicarse con" los } \\
\text { sujetos del estudio }\end{array}$ \\
\hline Se limita a responder. & Se limita a preguntar. \\
\hline & $\begin{array}{l}\text { Comunicación más horizontal...entre el } \\
\text { investigador y los investigados... mayor } \\
\text { naturalidad y habilidad de estudiar los } \\
\text { factores sociales en un escenario } \\
\text { natural. }\end{array}$ \\
\hline $\begin{array}{l}\text { Son débiles en términos de } \\
\text { validez interna - casi nunca } \\
\text { sabemos si miden lo que } \\
\text { quieren medir-, pero son fuertes } \\
\text { en validez externa, lo que } \\
\text { encuentran es generalizable a la }\end{array}$ & $\begin{array}{l}\text { Son fuertes en términos de validez } \\
\text { interna, pero son débiles en validez } \\
\text { externa, lo que encuentra no es } \\
\text { generalizable a la población. }\end{array}$ \\
\hline $\begin{array}{l}\text { Pregunta a los cualitativos: ¿Son } \\
\text { generalizables tus hallazgos? }\end{array}$ & $\begin{array}{l}\text { Pregunta a los cuantitativos : ¿Cuan } \\
\text { particularizables son los hallazgos?. }\end{array}$ \\
\hline
\end{tabular}

Fuente: Artículo sobre "Investigación Cuantitativa y Cualitativa" pág. 3

Autores: Pita Fernández, S., \& Pértegas Díaz, S. (2002)

Según Alvira "Ambas perspectivas han llevado a cabo un proceso convergente y de reconocimiento mutuo. La perspectiva cuantitativa ha aflojado en alguna medida el énfasis en la construcción de teorías a través de la modelización y simulación; la 
cualitativa ha seguido con su énfasis en la construcción y generación de teorías, aumentando el aspecto verificativo y confirmativo" (citado por Valles, 2000, p. 19).

Razón por la cual algunos autores plantean el uso combinado de métodos cuantitativos y cualitativos; como lo manifiestan Pita Fernández \& Pértegas Díaz "El empleo de ambos procedimientos cuantitativos y cualitativos en una investigación probablemente podría ayudar a corregir los sesgos propios de cada método" (2002 p. 2).

De este modo se plantea que la investigación debe realizarse desde un enfoque mixto, esto es reunir aspectos cualitativos y cuantitativos: se ha reconocido casi desde siempre la unicidad de la experiencia personal "lo cualitativo", pero sin desatender los aspectos comunes de las necesidades y los cuidados respectivos "lo cuantitativo" (García, J. C., \& Martínez, M. R., 1996).

Frente a los planteamientos y recomendaciones emitidas de trabajar con un enfoque mixto cuanti-cuali; surge a su vez, nuevos planteamientos de trabajar con metodologías alternativas que trascienden e integran lo cuantitativo y cualitativo.

Desde las perspectivas metodológicas de la complejidad en los diferentes procesos de investigación donde el sujeto juega un papel importante. Villasante (2006) indica que lo que hace científico a los resultados obtenidos consisten en una apuesta a una justificación metodológica pertinente para su comprobación/verificación.

Dentro de estas apuestas metodológicas se centran los diferentes procesos y estrategias de acción para la respectiva relación con los individuos a partir del ingenio y la capacidad de crear esquemas; así Tomas Villasante (2006) indica que los estilos creativos lo pueden ser a partir de la complejidad de la gente, pues que las personas seamos tan complejas, no nos parece una dificultad insalvable, sino una oportunidad.

Gómez, Rosa y Ruiz (2004) indican que el eje metodológico fundamental no está constituido por mecanismos de extracción de información como los que funcionan en encuestas, entrevistas de distinto formato, o incluso grupos de discusión, sino por herramientas de construcción colectiva de conocimiento al modo de talleres de creatividad. 
Alberich Nistal Tomás $(2008$, p.135) identifica a más de las dos grandes metodologías clásicas un tercer tipo de metodología denominada participativa que... "parte de la consideración de que el sujeto a investigar es el protagonista de la investigación y de que la finalidad de la investigación es la transformación social. Utilizando algunas técnicas propias de este tipo de metodología pero sin rechazar el uso de las técnicas cuantitativas y cualitativas".

Las metodologías participativas constituyen un conjunto de técnicas alternativas que promueven el diálogo entre el saber académico y el saber social (popular). Es decir tienen en cuenta la relación sujeto-sujeto, más allá de la relación tradicional entre sujeto-investigador y objeto de investigación-gente. Sin embargo, el saber académico también contiene saberes sociales y viceversa.

En las metodologías participativas "la población participa activamente con el investigador y los técnicos en el análisis de la realidad y en las acciones concretas para modificarla" (Navarrete, 2004, p. 294). El investigador es el que dinamiza y promueve el diálogo, el que plantea las preguntas, el que provoca la comunicación y la expresión en todas sus formas, para ello debe saber escuchar activamente los discursos y las acciones. El investigador no enseña lo que sabe sino que tiene presente que la interrogación y la recreación de saberes es una tarea inacabada permanente, cíclica y continua.

Las metodologías participativas "parten de tener en cuenta los diversos actores sociales que tienen intereses en los procesos de investigación o de intervención. Porque antes de plantearse técnicas de tipo cualitativo o cuantitativo, se plantean los problemas previos: ¿Quién manda aquí? ¿Cómo pueden contrarrestar los efectos negativos de los intereses en juego? No solo es cuestión de ética o de ideología, es cuestión de metodología básica la que planteamos"...La llamada "comunidad científica" no es un todo objetivo, sino una suerte de tendencias en disputa, por lo que quien promueve una investigación puede estar apoyando una $u$ otras tendencias de "poder" y de saber"' (Villasante, 2009, pp. 63 - 64).

Las metodologías participativas recuperan diversas técnicas de investigación como, por ejemplo, el grupo operativo, el mapa de actores, las entrevistas y grupos de discusión, las encuestas deliberativas, las asambleas participativas, entre otras. 
Villasante (2006, p. 400) menciona entre los tipos de metodologías participativas tradicionales "a la investigación-acción-participativa, praxeología y el socio-análisis. Pero también destaca aportaciones científicas, sociales y naturales como la "teoría de redes", "ecofeminismo", "ecología popular", "cibernética de 2 do. orden" o la "autopoiesis", "fractales", "simbiogénesis" y paradigmas de la "complejidad" que están detrás de estos razonamientos y que se han ido incorporando a la aplicación de las metodologías participativas concretamente desde la socio-praxis".

La Investigación-Acción-Participativa IAP Alberich N. Tomás (2008, p. 139) se define como "un método de estudio y acción que busca obtener resultados fiables y útiles para mejorar situaciones colectivas, basando la investigación en la participación de los propios colectivos a investigar, que así pasan de ser "objeto" de estudio a sujeto protagonista de la investigación, controlando e interactuando a lo largo del proceso investigador (diseño, fases, devolución, acciones, propuestas...) y necesitando una implicación y convivencia del investigador externo en la comunidad a estudiar".

La praxeología se caracteriza por el análisis de ciertas vivencias e implicaciones, y sigue luego con las reflexiones auto-críticas y críticas que entran en juego entre sí.....Pero la praxis no acaba e un diagnóstico sino en propuesta para la acción misma $y$ en las constantes reformulaciones que exige. Teniendo como esquema que las que las cosas y las ideas cambian cuando se cambian las condiciones de vida.

En el socio-análisis juega un papel fundamental el análisis de todo el contexto situacional y lingüístico. Los socio analistas se plantean como tarea el poder discernir y construir conocimientos útiles más emancipadores (Villasante, 2006).

La posición socio-praxica en cambio usa todas las metodologías cuantitativas, cualitativas y participativas desarrolladas hasta el momento pero no desde las metodologías y epistemes con las que suelen ser usadas, sino desde las posiciones implicativas y participativas. Más bien con una nueva visión ante la complejidad de los sistemas para construir colectivamente el conocimiento y la acción, es decir que se complementa lo cuantitativo que describe elementos, recorriendo por lo cualitativo que pretende encontrar las motivaciones de los comportamientos, de las frases, y la correlación entre lo que dice uno y otro actor para avanzar a un enfoque en que se 
construye con las voluntades de la comunidad en donde se quieren reconstruir relaciones multipartes/multiactores (Villasante, 2006).

En el siguiente gráfico se muestra a través del "multilema" la posición de metodologías dominantes: cuantitativa y cualitativa y el re-planteamiento a través de la utilización de metodologías alternativas: participativas.

Gráfico Nro. 1

REPLANTAMIENTO PARTICIPATIVO/ CUALI/CUANTI (PCC)

Fuente: Artículo sobre "la socio-praxis: un acoplamiento de metodologías implicativas" de Tomás R. Villasante

Autores: Elvis Orellana, Gabriela Álava

En el "multilema" se visualiza el re-plantamiento cuanti-cuali-parti que se propone utilizar. A través de un enfoque integrado que trasciende a través de la socio-praxis en la manera de indagar y recabar en la recolección de información.

Jesús Ibáñez concluye que: "un proceso concreto de investigación exige casi siempre integrar técnicas de las tres perspectivas: distributiva o cuantitativa, estructura o cualitativa y dialéctica o participativa" (citado por Valles, 2000, p. 22). Con lo cual, tanto Ibáñez como Valles dejan presente que en el terreno de la práctica investigadora es recomendable integrar las dimensiones analizadas, pero sin renunciar a la reflexión de 
una complejidad de una polémica metodológica y epistemológica cuantitativa y cualitativa.

\section{BIBLIOGRAFÍA}

Adamson, Gladys; Sapia, Pablo -ilustrador-. (2005). Psicología social para principiantes. Buenos Aires, Argentina: Editorial Era Naciente.

Alberich Nistal Tomás (2008). IAP, redes y mapas sociales: desde la investigación a la intervención social. Portularia: Revista de Trabajo Social, (8), 131-151.

Capra, Fritjof. (1992). El Punto Crucial. Ciencia, sociedad y cultura naciente. Buenos Aires, Argentina: Editorial \& Estaciones.

Capra, Frijtof. (2006). La trama de la vida. Una nueva perspectiva de los sistemas vivos. Barcelona, España: Editorial Anagrama.

Capra, Fritjof. (1992). El Punto Crucial. Ciencia, sociedad y cultura naciente. Buenos Aires, Argentina: Editorial \& Estaciones.

Estermann, Josef. (2009). Hacia una interversidad de saberes. ASAFTI. Recuperado de

http://www.asafti.org/site /index.php?

option=com content\&view=article\&id=42: hacia-unainterversidad-

saberes\&catid=11: culturas-ibero-americanas \&ltemid $=11$

García, J. C., \& Martínez, M. R. (1996). El debate investigación cualitativa frente a investigación cuantitativa. Enfermería clínica, 6(5), 213.

Gómez, A.; Rosa, M y Ruiz, E. (2004).Transformación organizacional y prestación de servicios. Investigación participativa en un centro de servicios sociales comunitarios "Sociología del trabajo 51" pp. 107-128.

Gómez, G. R., Flores, J. G., \& Jiménez, E. G. (1996). Metodología de la investigación cualitativa. Aljibe.

Navarrete, J. M. (2004). Sobre la investigación cualitativa. Nuevos conceptos y campos de desarrollo. Investigaciones Sociales, Lima, 8(13), 277-299.

Pita Fernández, S., \& Pértegas Díaz, S. (2002). Investigación cuantitativa y cualitativa. Cad Aten Primaria, 9, 76-8.

Reichardt, C. S., Cook, T. D., \& Martín, F. R. A. (1982). La perspectiva cualitativa y cuantitativa en las investigaciones sociales. Estudios de psicología, (11), 34-39. 
Santos, Boaventura de Sousa. (2010). La Refundación del Estado en América Latina. Perspectivas desde una epistemología del Sur. Quito, Ecuador: Editorial AbyaYala.

Valles, M. S. (2000). Técnicas cualitativas de investigación social. Síntesis Editorial.

Villasante, Tomás R. (2009, octubre). Metodologías para desbordar patriarcados y construir democracias participativas. En Raúl Zibechi (editor). Universidad en movimiento. Debates y memorias del $X$ Congreso Iberoamericano de Extensión Universitaria. Uruguay: Editorial Nordan. Argentina: Editorial El Colectivo.

Villasante, Tomás R. (2006). Desbordes Creativos. Estilos y estrategias para la transformación social. Madrid, España: Editorial Catarata.

Villasante, Tomás R. (2006). La socio-praxis: un acoplamiento de metodologías implicativas. Metodología de investigación social. LOM, Santiago de Chile, 379-408.

Villavicencio, Arturo. (2013). ¿Hacia dónde va el proyecto universitario de la revolución ciudadana?. Quito, Ecuador: Universidad Andina Simón Bolívar. Recuperado de https://www.google.com.ec/url?

$\mathrm{sa}=\mathrm{t} \& \mathrm{rct}=\mathrm{j} \& \mathrm{q}=\&$ esrc=s\&source=web\&cd=2\&cad=rja\&ved=0CDIQFjAB\&url=http\%3A

\%2F\%2Fwww.reaces.ec\%2Freaces\%2Findex.php\%3Foption\%3Dcom_docman

\%26task\%3Ddoc_download\%26gid\%3D58\%26ltemid\%3D55\&ei=oFjLUovNFYOgkAfyICICw\&usg=AFQjCNETvOI-YHSGbE7UsruXHAW-1unWiQ

Walsh, Catherine. (2009). Interculturalidad, Estado, Sociedad. Luchas (de) coloniales de nuestra época. Ecuador, Quito: Universidad Andina Simón Bolívar, Ecuador. AbyaYala Ediciones. 